08

\title{
Микроструктура переходных границ в многослойных Мо/Ве-системах
}

\author{
() Р.М. Смертин, ${ }^{1}$ В.Н. Полковников, ${ }^{1}$ Н.Н. Салащенко, ${ }^{1}$ Н.И. Чхало, ${ }^{1}$ П.А. Юнин, ${ }^{1}$ А.Л. Тригуб ${ }^{2}$ \\ ${ }^{1}$ Институт фоиики микроструктур РАН, \\ 607680 Нижний Новгород, Россия \\ ${ }^{2}$ Национальный исследовательский центр „Курчатовский институт“, \\ 123182 Москва, Россия \\ e-mail: smertin_ruslan@ipmras.ru
}

Поступило в Редакцию 22 апреля 2020 г.

В окончательной редакции 22 апреля 2020 г.

Принято к публикации 22 апреля 2020 г.

Методами рентгеновской рефлекто- и дифрактометрии и EXAFS-спектроскопии исследована микроструктура Мо/Ве многослойных периодических систем. Установлено, что в Мо/Ве-системе на границах образуются перемешанные зоны разного состава. На границе Мо-на-Ве - перемешанная зона, схожая по составу с $\mathrm{MoBe}_{22}$, а на границе Ве-на-Мо - с $\mathrm{MoBe}_{2}$. В результате термического отжига в течение $1 \mathrm{~h}$ структура переходных границ в многослойной системе остается стабильной. При дальнейшем отжиге происходят диффузионные процессы, которые приводят к образованию другого соединения на границе раздела - $\mathrm{MoBe}_{2}$ вместо $\mathrm{MoBe}_{22}$, однако период структуры при этом остается неизменным. Такое поведение объясняет рост коэффициента отражения Мо/Ве-зеркал после отжига в течение $1 \mathrm{~h}$ и дальнейшее падение коэффициента отражения при большем времени отжига.

Ключевые слова: многослойные зеркала, EXAFS-спектроскопия, межслоевые области, термическая стабильность, рентгеновское излучение.

DOI: 10.21883/JTF.2020.11.49979.142-20

\section{Введение}

Интерес к многослойным зеркалам (МЗ) на основе Мо/Ве пробудился в 1990-х годах и был связан с проекционной литографией в ВУФ-диапазоне [1-4]. Выбор осуществлялся между рабочими длинами волн 11.2 и $13.5 \mathrm{~nm}$. В этот период уделялось большое внимание изучению зеркал $\mathrm{Mo} / \mathrm{Be}$, оптимизированных на окрестность длины волны $11.2 \mathrm{~nm}[5,6]$. При том, что на длине волны $11.3 \mathrm{~nm}$ получено рекордное пиковое значение коэффициента отражения 70.2\% [6], приоритет был отдан литографии с рабочей длиной волны $13.5 \mathrm{~nm}$ и оптике на основе кремния. Следует отметить, что зеркала $\mathrm{Mo} / \mathrm{Si}$ обладали в полтора раза более широкой спектральной шириной пика отражения на половине высоты при чуть меньшем пиковом значении коэффициента отражения.

В 2000-х годах продолжались работы по изучению возможностей литографии с рабочей длиной волны короче $13.5 \mathrm{~nm}$. Достаточно развитая на сегодняшний день технология $13.5 \mathrm{~nm}$ позволяет без возрастающей сложности достигать разрешения порядка $10 \mathrm{~nm}$ [7]. Дальнейшее снижение этой величины связано с рядом технических сложностей. В то же время переход к более короткой длине волны $(11 \mathrm{~nm})$ автоматически на 20\% повышает разрешение проекционного объектива при фактическом сохранении принципиальных составляющих отработанного технологического процесса. Дополнительный плюс связан с возможностью использования газовой мишени (ксеноновая плазма) взамен оптимальной для $13.5 \mathrm{~nm}$ оловянной. Такая мишень не дает тех загрязнений оптики, источником которых служит олово.

Оценки показывают, что пропускная способность системы из 10 зеркал, оптимизированных на $\lambda=11.2 \mathrm{~nm}$ $(\mathrm{Mo} / \mathrm{Be}, \mathrm{Ru} / \mathrm{Be})$, может быть вдвое выше, чем такая же величина для зеркал $\mathrm{Mo} / \mathrm{Si}$ на $13.5 \mathrm{~nm}$, что с учетом коэффициента конверсии соответствующих источников излучения говорит о предпочтительности развития технологии литографии с рабочей длиной волны $11.2 \mathrm{~nm}$ [8]. Однако разработке многозеркальных проекционных схем должно предшествовать изучение возможностей создания высокоэффективных зеркал нормального падения, оптимизированных на $\lambda=11.2 \mathrm{~nm}$.

Прекращенные в конце 1990-х годов работы по изучению свойств бериллий-содержащих МЗ были возобновлены в 2014 г. в ИФМ РАН, где создана уникальная бериллиевая лаборатория [9]. В ходе исследований на длине волны $11.3 \mathrm{~nm}$ для М3 на основе Mo/Ве был получен коэффициент отражения 70.25\% [10], что совпадает с результатами, ранее опубликованными в [7].

Следует отметить, что теоретическим пределом для $\mathrm{Mo} / \mathrm{Be}$ на этой длине волны является 76\%. Основной причиной несоответствия теоретического предела достигнутым на практике значениям являются уширенные переходные области на границах раздела между материалами структуры. Внедрение методики барьерных слоев [8] формально не привело к улучшению отражательной способности МЗ. Однако в этом исследовании было показано, что кремниевый буферный слой приводит к 
сглаживающему эффекту, т.е. к уменьшению величины межслоевой шероховатости.

Изучение этого эффекта было продолжено на примере зеркал $\mathrm{Mo} / \mathrm{Be} / \mathrm{Si}$, оптимизированных на окрестность длины волны $13.5 \mathrm{~nm}$. Экспериментально достигнуты значения пикового коэффициента отражения $R=71.9 \%$ на $13.5 \mathrm{~nm}$ и $72.8 \%$ на $12.9 \mathrm{~nm}$ [11]. Полуширина спектральной кривой отражения на $13.5 \mathrm{~nm}$ составила около $0.52 \mathrm{~nm}$, что практически не уступает классическим $\mathrm{Mo} / \mathrm{Si}$-зеркалам, полуширина которых составляет около $0.54 \mathrm{~nm}$.

Важным преимуществом M3 Mo/Be над Mo/Si является их высокая термическая стойкость. В работах $[12,13]$ показано, что вплоть до температур $350^{\circ} \mathrm{C}$ М3 Mo/Be сохраняют отражательные свойства. Более того, при определенных режимах отжига наблюдается некоторое увеличение коэффициента отражения (порядка абсолютного $1 \%$ ).

Тем не менее и в данном случае теоретический предел отражения не достигнут. Проблема связана не только с наличием геометрической шероховатости на границах раздела, но также с возможным образованием в пограничных областях химических соединений материалов, силицидов и бериллидов. И если вопрос образования силицидов молибдена уже изучался [14-17], то изучение бериллидов и вообще структуры границ в тонкопленочных периодических структурах на основе Мо/Ве практически не проводилось.

Единственной работой, затрагивающей вопрос структуры границ в многослойных структурах $\mathrm{Mo} / \mathrm{Be}$, является [18]. Здесь методами фотоэлектронной спектроскопии была исследована микроструктура переходных границ в системе $\mathrm{Mo} / \mathrm{Be}$ с разным количеством периодов от одного до ста. При этом период структуры и соотношение толщин слоев были близки к значениям, обеспечивающим максимальное значение коэффициента отражения в окрестности длины волны $11.4 \mathrm{~nm}$. Было установлено образование разных соединений Мо и Ве на разных границах. Так, на границе Мо-на-Ве образуется перемешанная зона, моделируемая как $\mathrm{MoBe}_{1.0-1.6}$, а на границе Ве-на-Мо - как $\mathrm{MoBe}_{4.0-5.0}$. Однако, исходя из данных фазовой диаграммы Мо-Ве-системы [19,20], таких соединений нет. Данные [20] объясняются тем, что в ходе расчета учитывалось среднее количество атомов, находящихся в переходной области. Толщины соединений, исходя из моделирования, составляют по $1 \mathrm{~nm}$ на каждой границе. Однако, исходя из данных [10], ширины переходных границ имеют другие значения. Граница Мона-Ве порядка $0.7 \mathrm{~nm}$, Be-на-Мо - порядка $0.35 \mathrm{~nm}$.

Эти противоречия, а также важность установления подробностей эволюции структуры пограничных областей при отжиге $\mathrm{Mo} / \mathrm{Be}$, делают необходимыми дальнейшие исследования. Настоящая работа посвящена изучению структуры пограничных областей М3 Mo/Be (отожженных и неотоженных) методами рефлектометрии, дифрактометрии и EXAFS-спектроскопии.

\section{1. Методика эксперимента}

M3 Mo/Ве изготавливались методом магнетронного напыления при постоянном токе $(I(\mathrm{Mo})=0.2 \mathrm{~A}$, $I(\mathrm{Be})=0.9 \mathrm{~A}$, напряжения около $300 \mathrm{~V})$ в среде $\mathrm{Ar}$ при давлении газа $\sim 0.2 \mathrm{~Pa}$. Зеркала осаждались на гладкие (величина среднеквадратичной микрошероховатости $\sim 0.2 \mathrm{~nm})$ кремниевые пластины. Структурные параметры многослойных систем определялись с использованием расширенной модели по данным малоугловой рентгеновской рефлектометрии (длина волны $0.154 \mathrm{~nm}$ ). Подробнее о методах синтеза и восстановления параметров структур по данным рентгеновской дифракции можно прочитать в $[9,21]$.

В ходе экспериментов изучались периодические структуры Мо/Ве различных типов. Толщины слоев Мо в них составляли: $\sim 0.2, \sim 0.6, \sim 1$ и $\sim 2.3 \mathrm{~nm}$. Количество периодов выбиралось таким, чтобы общая толщина Мо в структурах составляла $\sim 100 \mathrm{~nm}$. Толщина слоев Ве во всех структурах оставалась постоянной и составляла $\sim 3.5 \mathrm{~nm}$. Также для исследований была изготовлена пленка Мо толщиной $\sim 100 \mathrm{~nm}$. Точность определения толщины составляет $1 \%$.

Структуры каждого типа изготовлялись в количестве трех экземпляров. Одна сохранялась в исходном виде. Две другие подвергались отжигу в течение 1 и $4 \mathrm{~h}$ соответственно. Отжиг структур проводился в вакуумной печи при давлении $6 \cdot 10^{-5} \mathrm{~Pa}$ и при $T=280^{\circ} \mathrm{C}$. Эта величина соответствует значению, при котором происходит увеличение коэффициента отражения при отжиге в течение $1 \mathrm{~h} \mathrm{[12].} \mathrm{Температура} \mathrm{контролирова-}$ лась термопарой хромель-алюмель с точностью $\pm 5^{\circ} \mathrm{C}$. Время нагрева и охлаждения не учитывалось.

Для определения степени кристалличности слоев и наличия соединений бериллия и молибдена (бериллидов молибдена) в Мо/Ве-структурах применялась широкоугловая рентгеновская дифракция с использованием дифрактометра Bruker D8 Discover. Измерение спектров XANES и EXAFS вблизи $K$-края поглощения Мо выполнено на станции СТМ Курчатовского центра синхротронного излучения. Энергетическое разрешение $\Delta E / E \sim 2 E-4$, размер пучка на образце $3 \times 1 \mathrm{~mm}$, монохроматор - прорезной монокристалл кремния с отражающей поверхностью (220), для детектирования флуоресцентного излучения использовался SDD-детектор АМРТЕК. Образец стоял перпендикулярно излучению. Излучение проходит сквозь исследуемый образец. После образца излучение проходит ионизационную камеру, эталон и следующую ионизационную камеру. В качестве эталона использовался прессованный порошок чистого Мо. Спектр поглощения исследуемого образца определяется по выходу флуоресценции, а эталона - исходя из разницы излучения в двух ионизационных камерах до и после прохождения эталона. Подробнее о схеме и проведении EXAFS-эксперимента можно найти в [22,23].

Определение структурных параметров из спектров EXAFS выполнено путем подгонки расчетных спектров 

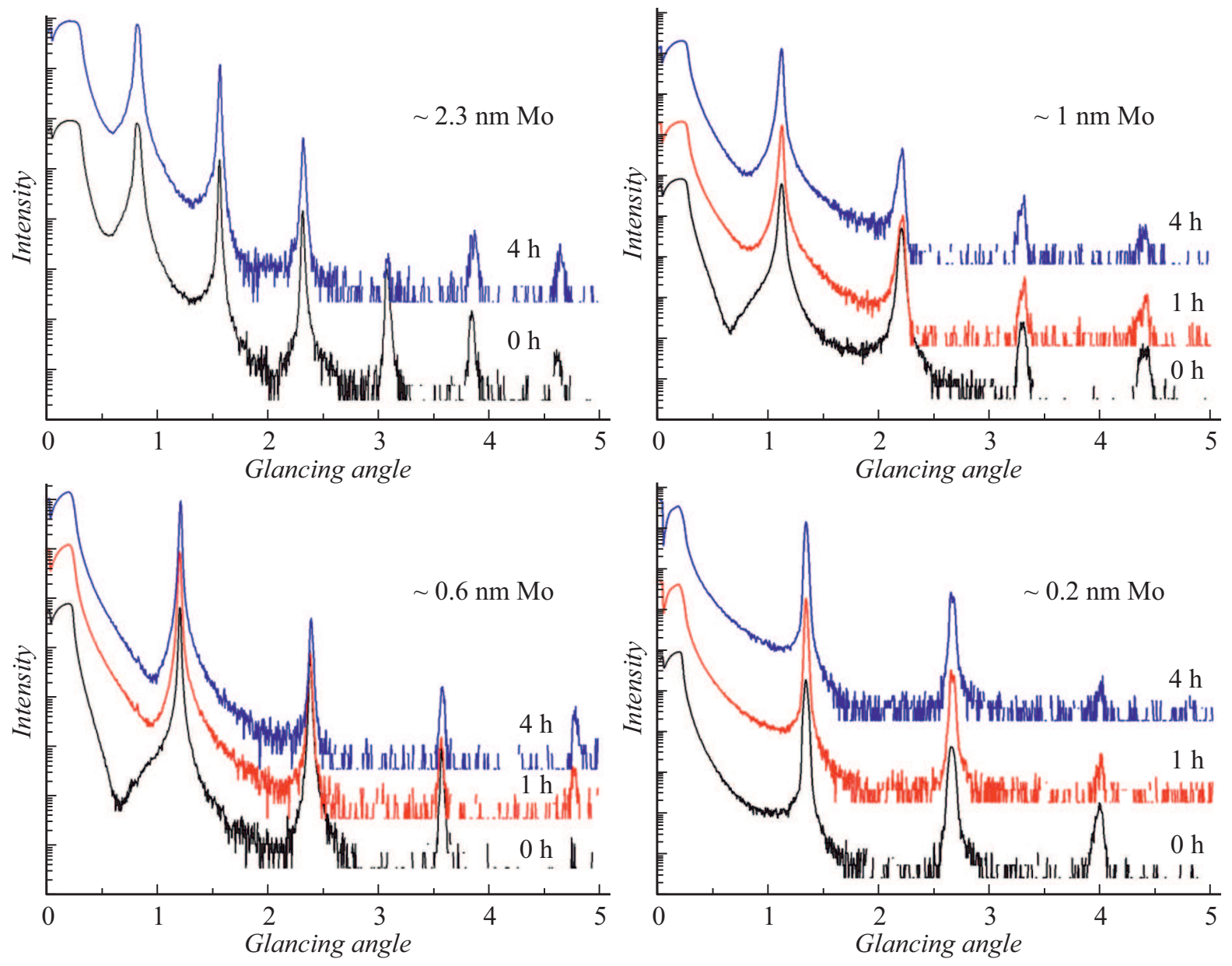

Рис. 1. Малоугловая рентгеновская рефлектометрия $(\lambda=0.154 \mathrm{~nm})$ от многослойных периодических систем Мо/Ве с разными толщинами Мо в структуре в исходном состоянии и после отжига.

к экспериментальным. Для этого использовался пакет программ IFFEFIT [24]. После стандартной процедуры вычитания предкраевой фоновой кривой, нормализации спектра и выделения осциллирующей части спектра, путем варьирование межатомных расстояний, координационных чисел и параметров Дебая-Валлера выполнена подгонка расчетного спектра к экспериментальному. Параметры, необходимые для расчета EXAFS-спектра (длина свободного пробега фотоэлектрона, амплитуда рассеяния и величина фазового сдвига при рассеянии фотоэлектрона на атомах окружения)? рассчитаны программой FEFF6 [25].

Теоретические расчеты XANES-спектров, измеренных на $K$-крае Мо, выполнены методом конечных разностей (FDM), как это реализовано в программе FDMNES [26,27]. Выполнен самосогласованный расчет электронной плотности с обменно-корреляционным потенциалом в приближении локальной плотности [28]. Плотность электронных состояний возбужденного атома рассчитана с учетом полной экранировки дырочного состояния. Расчет выполнен для атомных кластеров радиуса $5 \AA$, построенных на основании кристаллогра- фических данных для фаз $\mathrm{MoBe}_{2}, \mathrm{MoBe}_{12}$ и $\mathrm{MoBe}_{22}$ [29]. Расчет спектра поглощения проводится путем свертки плотности незанятых электронных состояний с кривой Лоренца. При этом наилучшее согласие расчета с экспериментом достигается, когда ширина кривой Лоренца зависит от энергии, как арктангенс [30].

\section{2. Экспериментальные результаты}

По результатам малоугловой рентгеновской рефлектометрии были определены периоды многослойных структур и соотношения толщин материалов. Из рис. 1 видно, что многослойные структуры Мо/Ве обладают хорошей периодичностью даже при толщинах слоев Мо $\sim 0.2 \mathrm{~nm}$. Периоды всех структур не меняются при отжиге. Это говорит о высокой термической стойкости многослойной системы Мо/Be.

Как видно из XANES- и EXAFS-спектров (рис. 2), положение осцилляций и положение края поглощения у пленки Мо и эталона Мо совпадают, однако интенсивность пиков в спектрах и в функции радиального 


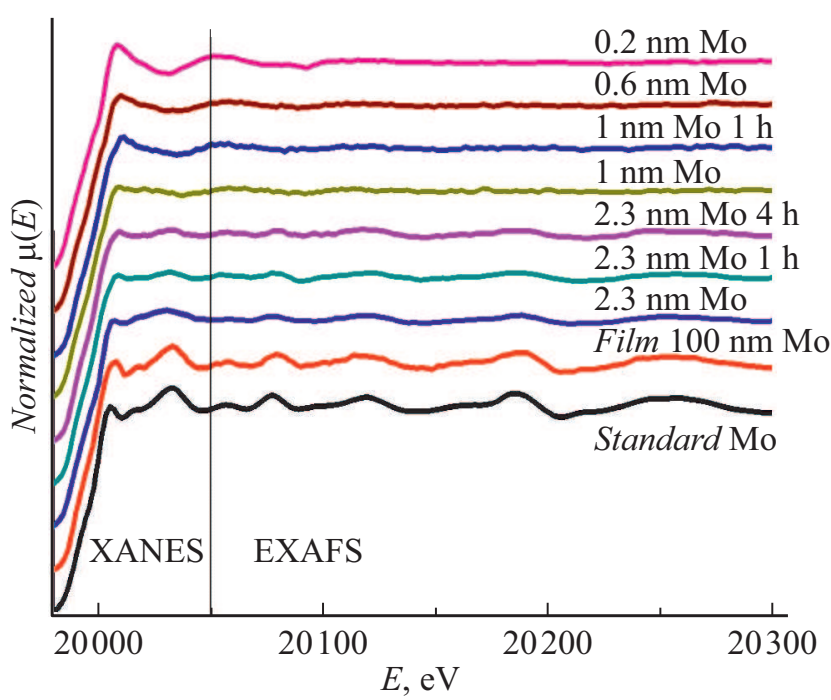

Pис. 2. XANES- и EXAFS-спектры многослойных периодических систем $\mathrm{Mo} / \mathrm{Be}$ с разной толщиной слоя Мо $(0.2,0.6,1$ и $2.3 \mathrm{~nm} \mathrm{Mo)} \mathrm{в} \mathrm{исходном} \mathrm{состоянии} \mathrm{и} \mathrm{после} \mathrm{отжига,} \mathrm{тонкой}$ пленки Мо, толщиной $100 \mathrm{~nm}$ и эталона Мо.

распределения (рис. 3) ниже, чем у эталона, что говорит о наличие дефектов в пленке Мо.

Спектры от многослойной системы $\mathrm{Mo} / \mathrm{Be}$ с толщиной Мо $\sim 2.3 \mathrm{~nm}$ имеют близкие положения осцилляций с таковыми у пленки Мо и эталона (рис. 2,3,a). Положение края поглощения смещено на $0.7 \mathrm{eV}$ относительно эталонного значения, что указывает на наличие дополнительной фазы в Мо-слое. Положение пиков в функции радиального распределения (рис. $3, b$ ) близко к положению таковых в пленке, а после термическо- го отжига смещается в сторону эталонного Мо. Пик, описывающий первую координационную сферу, имеет несимметричный вид с плечом, расположенным со стороны меньших расстояний. Как видно из рис. $3, b$, аналогичная асимметрия пика наблюдается и в пленке Мо, и в эталоне Мо. При разложении пика, описывающего первую координационную сферу, на две компоненты было установлено, что второй пик, характеризующий левое плечо, имеет интенсивность ниже фонового значения, что не позволяет уверенно говорить о присутствии бериллидной фазы в системе $\mathrm{Mo} / \mathrm{Be}$ с толщиной Мо в структуре $\sim 2.3 \mathrm{~nm}$.

Анализ XANES- и EXAFS-спектров многослойных систем $\mathrm{Mo} / \mathrm{Be}$ с толщинами слоев Мо $\sim 1, \sim 0.6$ и $\sim 0.2 \mathrm{~nm}$ обнаруживает несоответствие XANESструктуры спектру как эталонного Мо, так и толстой пленки Мо, а также отсутствие осцилляций в EXAFSобласти (рис. 2). В спектрах XANES систем $\mathrm{Mo} / \mathrm{Be}$ наблюдается сдвиг края поглощения в сторону меньших энергий относительно эталонного Мо. Сдвиг тем сильнее, чем меньше толщина Мо-слоя: $1 \mathrm{eV}$ для системы $\mathrm{Mo} / \mathrm{Be}$ с толщиной Mo $\sim 1 \mathrm{~nm}, 1.1 \mathrm{eV}$ для $\sim 0.6 \mathrm{~nm}$ Мо и $2.1 \mathrm{eV}$ для $\sim 0.2 \mathrm{~nm}$ Мо. Исходя из обнаруженного сдвига края поглощения в XANES-спектрах систем $\mathrm{Mo} / \mathrm{Be}$, можно предположить образование в них новой фазы - соединения Мо с Ве. Однако сопоставление положения краев поглощения в спектрах XANES образцов с разной толщиной Мо в структуре и теоретически рассчитанных спектров бериллидов (рис. 4) указывает на то, что спектры образцов с толщинами слоя Мо $\sim 0.6 \mathrm{~nm}$ (рис. $4, b)$ и $\sim 1 \mathrm{~nm}$ (рис. $4, a)$ не соответствуют спектрам ни одного из теоретически рассчитанных бериллидов как в исходном состоянии, так и после отжига. $a$

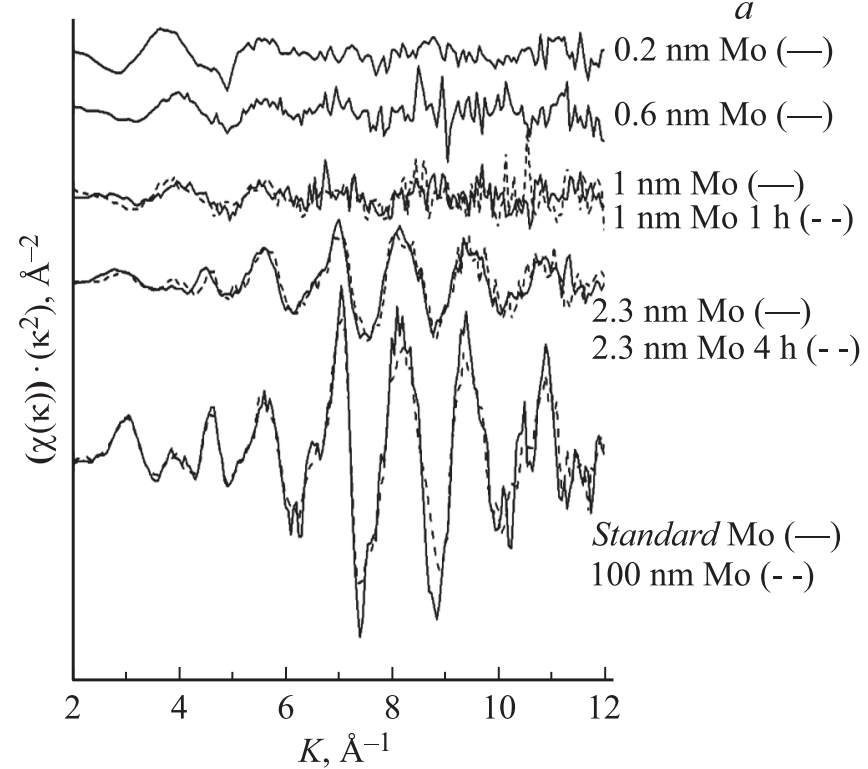

$b$

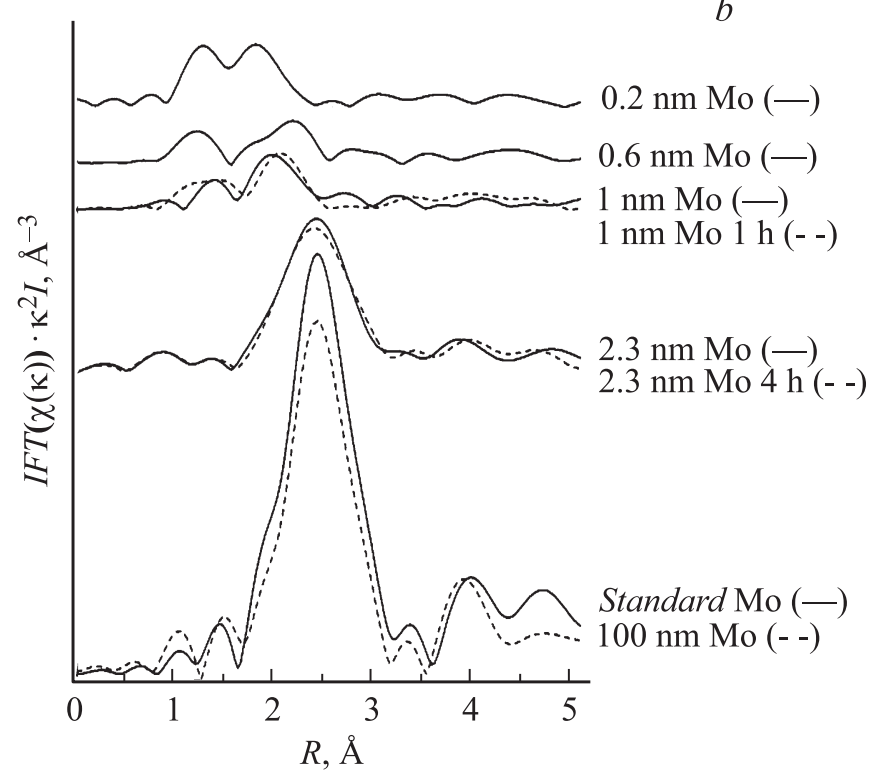

Рис. 3. Спектры EXAFS $(a)$ и функция радиального распределения атомов $(b)$ в многослойных периодических системах Мо/Ве $(0.2,0.6,1$ и $2.3 \mathrm{~nm}$ Мо) в исходном состоянии и после отжига, тонкой пленки Мо, толщиной $100 \mathrm{~nm}$ и эталона Мо. 

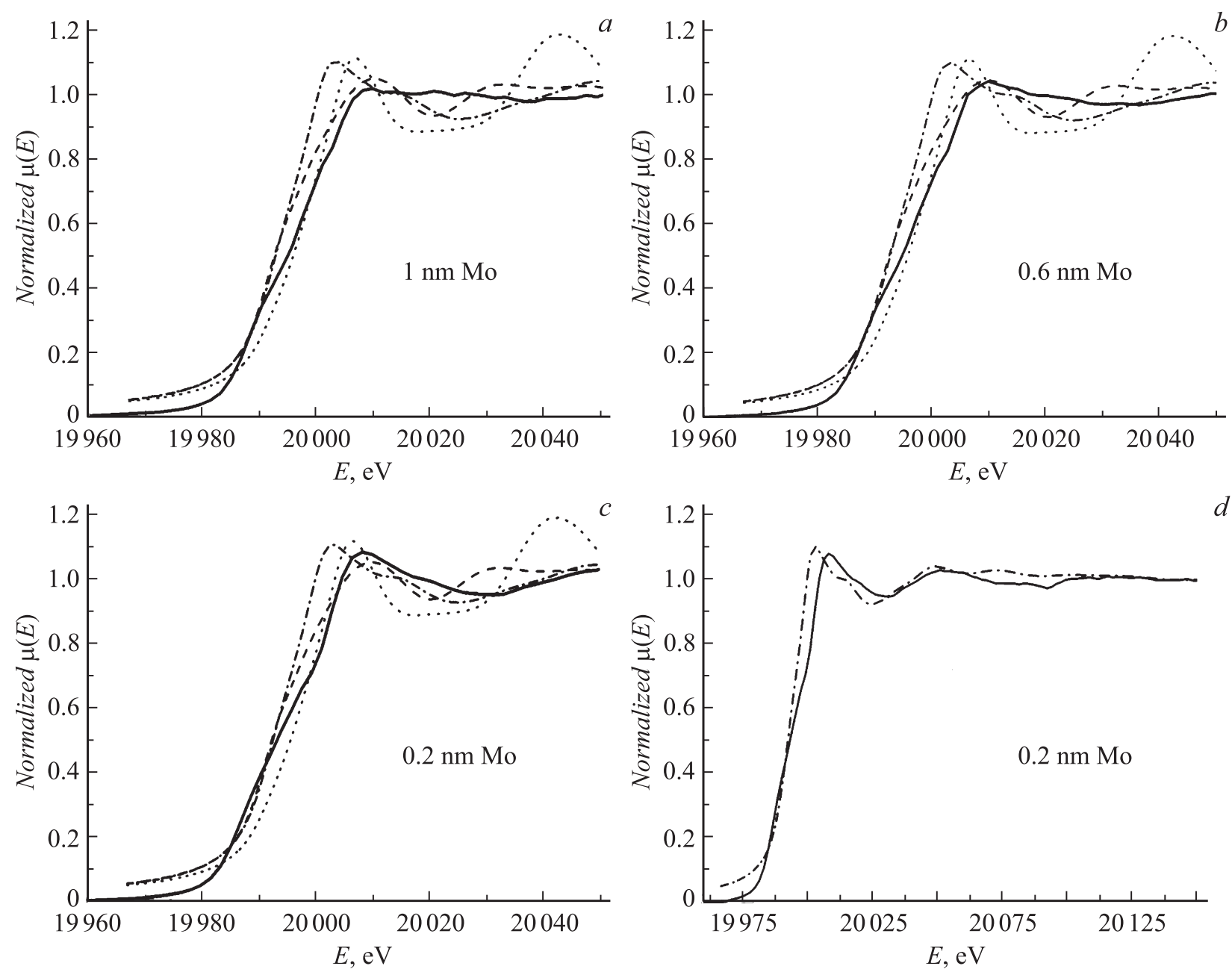

Рис. 4. Сравнение XANES-спектров от некоторых образцов Мо/Ве с разными толщинами Мо в структуре ( $a-1 \mathrm{~nm}$ Мо, $b-0.6 \mathrm{~nm} \mathrm{Mo,} c$ и $d-0.2 \mathrm{~nm} \mathrm{Mo)} \mathrm{с} \mathrm{теоретическими} \mathrm{XANES-спектрами} \mathrm{бериллидов} \mathrm{молибдена:} \mathrm{MoBe}_{2}(---), \mathrm{MoBe}_{12}(\cdots)$, $\mathrm{MoBe}_{22}(-\cdot \cdot)$.

Также положение края поглощения у данных образцов не соответствует положению таковых у теоретически рассчитанных спектров бериллидов.

В свою очередь, XANES-спектр образца Мо/Be с толщиной Мо $\sim 0.2 \mathrm{~nm}$ по форме (числу деталей и соотношению их интенсивностей) более всего похож на теоретически рассчитанный XANES-спектр $\mathrm{MoBe}_{22}$ (рис. $4, c, d)$. В то же время, как и в случае других образцов, положение края поглощения XANES-спектра этого образца не соответствует ни одному из положений краев поглощения рассчитанных XANES-спектров рассмотренных бериллидов. Данная информация дает основание предполагать, что в многослойной системе Мо/Ве с тонким слоем Мо $\sim 0.2 \mathrm{~nm}$ в структуре образуется соединение Мо с Ве, схожее по составу с $\mathrm{MoBe}_{22}$. Предположительно при увеличении толщины дополнительно образуется другое соединение Мо с Ве, которое в совокупности с образованным соединением, схожим с $\mathrm{MoBe}_{22}$, дает средний XANES-спектр, не поддающийся анализу.
В результате подгонки экспериментальных EXAFSспектров и функций радиального распределения атомов (рис. 5) были установлены радиусы координационных сфер и координационные числа (табл. 1). В табл. 2 представлены расчетные значения радиусов координационных сфер и координационных чисел Мо и бериллидов молибдена.

Радиусы первой координационной сферы в системе Мо/Ве с толщинами Мо $\sim 0.2, \sim 0.6$ и $\sim 1 \mathrm{~nm}$ имеют значения, близкие к значениям в трех бериллидах: $\mathrm{MoBe}_{2}, \mathrm{MoBe}_{12}$ и $\mathrm{MoBe}_{22}$. Однако, как указывалось ранее, только структура с толщиной Мо $\sim 0.2 \mathrm{~nm}$ имеет схожесть в своем виде с бериллидом $\mathrm{MoBe}_{22}$, что позволяет предположить его образование. Однозначно установить состав перемешанных зон у других толщин невозможно.

При больших толщинах слоя Мо $(\sim 2.3 \mathrm{~nm})$ в структуре $\mathrm{Mo/Be} \mathrm{радиусы} \mathrm{координационных} \mathrm{сфер} \mathrm{близки}$ к таковым у пленки Мо. Координационное число в системе в исходном состоянии ниже, чем в чистой 

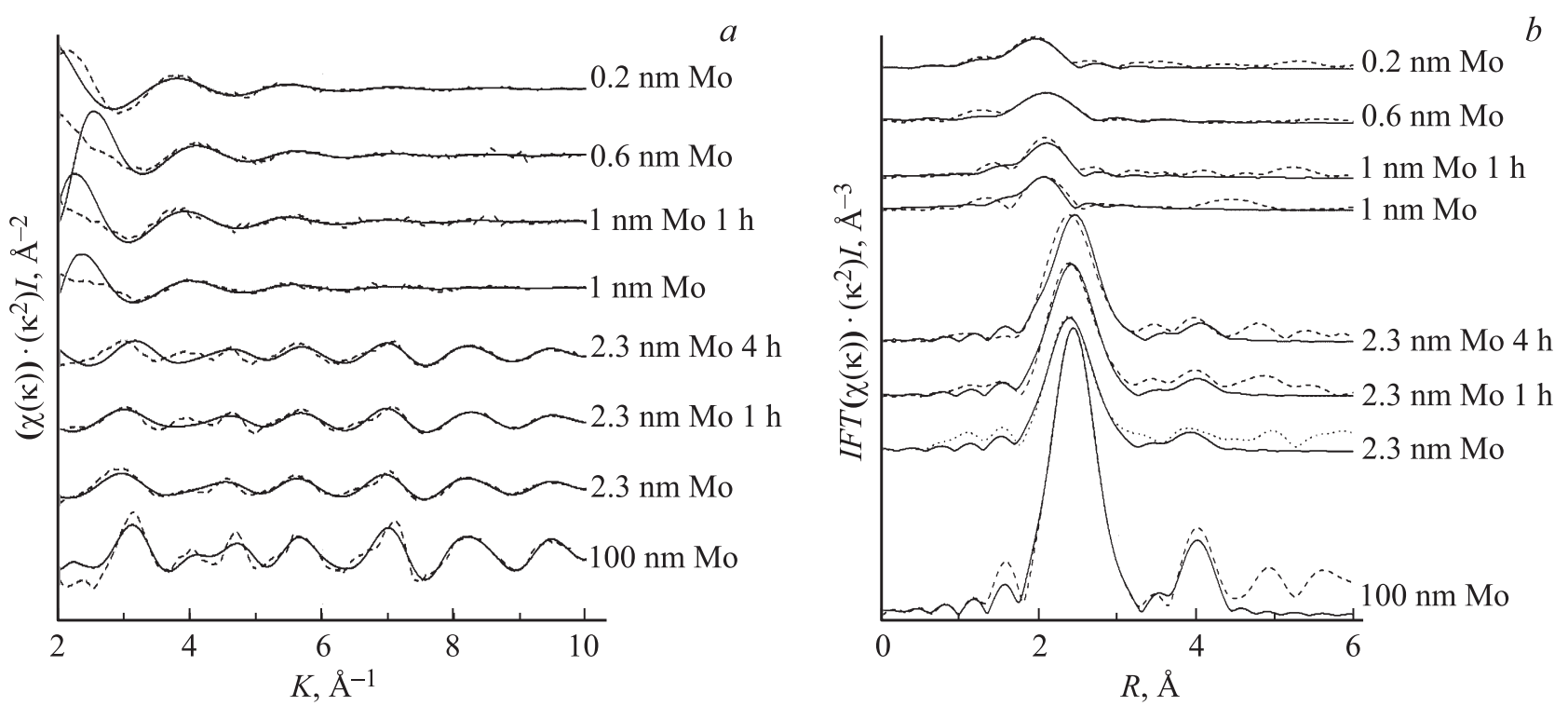

Рис. 5. Экспериментальные (--) и подгоночные (-) EXAFS-спектры $(a)$ и функции радиального распределения $(b)$ от многослойных периодических систем Мо/Ве в исходном состоянии и после отжига и тонкой пленки Мо толщиной $100 \mathrm{~nm}$.

Таблица 1. Межатомные расстояния и координационные числа, полученные из EXAFS-эксперимента

\begin{tabular}{|c|c|c|c|c|}
\hline $\begin{array}{c}\text { Толщина Мо } \\
\text { в структуре } \\
\text { и время отжига }\end{array}$ & Связь & $R, \AA$ & $\begin{array}{c}\text { Координационное } \\
\text { число }\end{array}$ & $\sigma^{2}, \AA^{2}$ \\
\hline $0.2 \mathrm{~nm}$ & Mo-Be & 2.54 & 4.0 & 0.004 \\
\hline $0.6 \mathrm{~nm}$ & Mo-Be & 2.60 & 3.5 & 0.003 \\
\hline $1 \mathrm{~nm}$ & Mo-Be & 2.60 & 2.7 & 0.003 \\
\hline $1 \mathrm{~nm} 1 \mathrm{~h}$ & Mo-Be & 2.61 & 3.8 & 0.003 \\
\hline \multirow{3}{*}{$2.3 \mathrm{~nm}$} & Mo-Mo & 2.69 & 3.4 & 0.005 \\
\hline & Mo-Mo & 3.11 & 2.4 & 0.004 \\
\hline & Mo-Mo & 4.36 & 2.4 & 0.006 \\
\hline \multirow{3}{*}{$2.3 \mathrm{~nm} 1 \mathrm{~h}$} & Mo-Mo & 2.69 & 3.2 & 0.005 \\
\hline & Mo-Mo & 3.10 & 1.6 & 0.005 \\
\hline & Mo-Mo & 4.40 & 2.8 & 0.004 \\
\hline \multirow{3}{*}{$2.3 \mathrm{~nm} 4 \mathrm{~h}$} & Mo-Mo & 2.72 & 3.4 & 0.005 \\
\hline & Mo-Mo & 3.13 & 2.3 & 0.005 \\
\hline & Mo-Mo & 4.42 & 3.3 & 0.008 \\
\hline \multirow{3}{*}{ Пленка 100 nm } & Mo-Mo & 2.71 & 5.9 & 0.004 \\
\hline & Mo-Mo & 3.11 & 5.1 & 0.008 \\
\hline & Mo-Mo & 4.40 & 12.0 & 0.004 \\
\hline
\end{tabular}

пленке Мо, что говорит о наличии большего числа дефектов в тонком слое, чем в толстой пленке. После отжига радиусы координационных сфер приближаются к
Таблица 2. Межатомные расстояния и координационные числа для кристалла молибдена и бериллидов молибдена

\begin{tabular}{|c|c|c|c|}
\hline Фаза & Связь & $R, \AA$ & $\begin{array}{c}\text { Координационное } \\
\text { число }\end{array}$ \\
\hline \multirow{3}{*}{ Mo } & Mo-Mo & 2.73 & 8 \\
\hline & Mo-Mo & 3.15 & 6 \\
\hline & Mo-Mo & 4.45 & 12 \\
\hline \multirow{4}{*}{$\mathrm{MoBe}_{2}$} & Mo-Be & 2.55 & 3 \\
\hline & Mo-Be & 2.59 & 3 \\
\hline & Mo-Be & 2.64 & 6 \\
\hline & Mo-Mo & 2.67 & 3 \\
\hline \multirow{5}{*}{$\mathrm{MoBe}_{12}$} & Mo-Be & 2.54 & 4 \\
\hline & Mo-Be & 2.61 & 8 \\
\hline & Mo-Be & 2.77 & 8 \\
\hline & Mo-Mo & 4.08 & 8 \\
\hline & Mo-Mo & 4.23 & 2 \\
\hline $\mathrm{MoBe}_{22}$ & $\begin{array}{l}\text { Mo-Be } \\
\text { Mo-Be } \\
\text { Mo-Be } \\
\text { Mo-Be }\end{array}$ & $\begin{array}{l}2.49 \\
2.52 \\
4.21 \\
4.29\end{array}$ & $\begin{array}{r}12 \\
4 \\
6 \\
24\end{array}$ \\
\hline
\end{tabular}

эталонным значениям, а также увеличиваются координационные числа, что говорит об уменьшении количества дефектов и увеличении дальнего порядка. Однако координационные числа у отожженных образцов меньше, чем в чистой пленке Мо. Наличие координационных 
сфер, соответствующих связям Мо-Ве в системе Мо/Ве с толщиной Мо $\sim 2.3 \mathrm{~nm}$ в структуре, установить не удалось, несмотря на то, что данные связи присутствуют в образцах с меньшей толщиной Мо в структуре.

На рис. 6-8 представлены картины рентгеновского фазового анализа от образцов в исходном состоянии и после термического отжига.

$\mathrm{У}$ многослойной системы $\mathrm{Mo} / \mathrm{Be}$ с толщиной Мо в структуре $\sim 0.2 \mathrm{~nm}$ наблюдаются рефлексы только от $\mathrm{MoBe}_{22}$ (рис. 6). Термический отжиг в течение одного часа не приводит к каким-либо изменениям в спектре, а после термического отжига в течение $4 \mathrm{~h}$ пик $\mathrm{MoBe}_{22}$ усиливается. Это свидетельствует об увеличении ширины переходной области за счет бериллида. Рефлекса от Мо в структуре не наблюдается, что в совокупности с данными EXAFS-эксперимента позволяет установить образование перемешанной зоны, схожей с $\mathrm{MoBe}_{22}$.

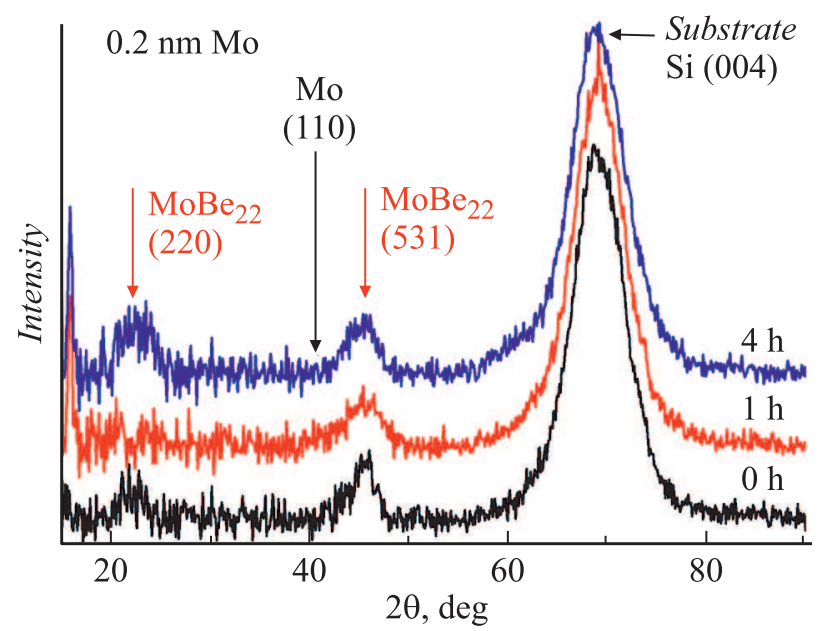

Рис. 6. Рентгеновский фазовый анализ $(\lambda=0.154 \mathrm{~nm})$ многослойной периодической структуры $\mathrm{Mo} / \mathrm{Be}$ с толщиной Мо в структуре $\sim 0.2 \mathrm{~nm}$.

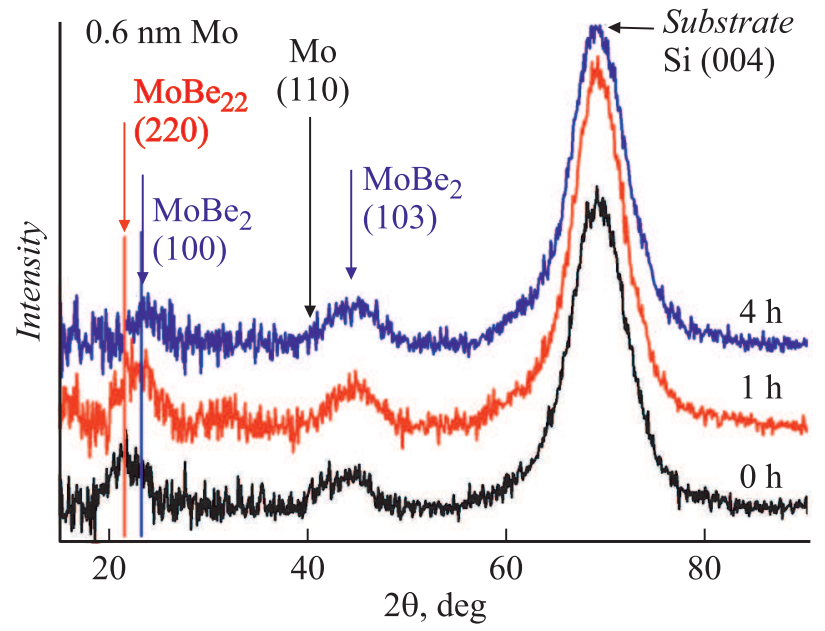

Рис. 7. Рентгеновский фазовый анализ $(\lambda=0.154 \mathrm{~nm})$ многослойной периодической структуры $\mathrm{Mo} / \mathrm{Be} \mathrm{c}$ толщиной Мо в структуре $\sim 0.6 \mathrm{~nm}$.

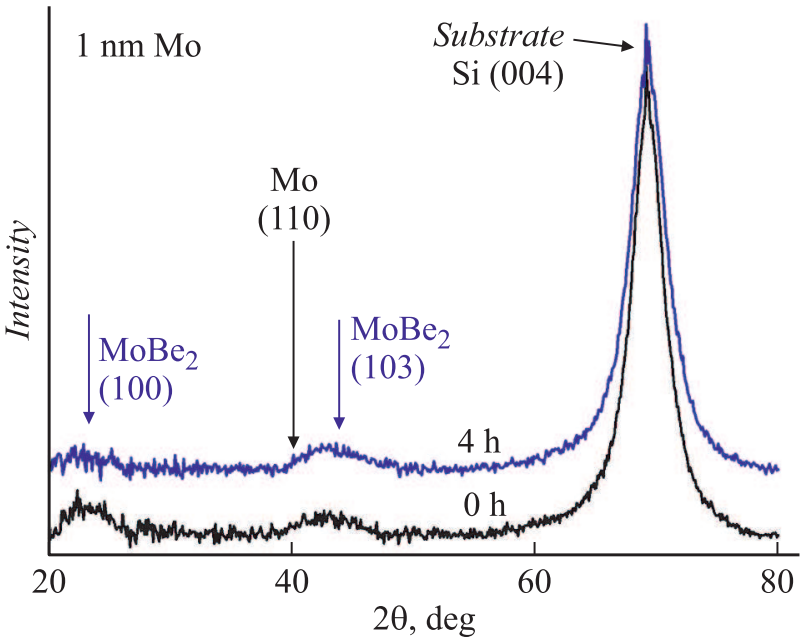

Рис. 8. Рентгеновский фазовый анализ $(\lambda=0.154 \mathrm{~nm})$ многослойной периодической структуры $\mathrm{Mo} / \mathrm{Be}$ с толщиной Мо в структуре $\sim 1 \mathrm{~nm}$.

$\mathrm{y}$ многослойной системы $\mathrm{Mo} / \mathrm{Be}$ с толщиной $\mathrm{Mo} \mathrm{в}$ структуре $\sim 0.6 \mathrm{~nm}$ в исходном состоянии и после термического отжига в течение $1 \mathrm{~h}$ наблюдаются рефлексы как от $\mathrm{MoBe}_{22}$, так и от $\mathrm{MoBe}_{2}$ (рис. 7), а после отжига в течение $4 \mathrm{~h}$ рефлекс от $\mathrm{MoBe}_{22}$ пропадает и появляется второй рефлекс $\mathrm{MoBe}_{2}$. Это означает, что в результате диффузионного процесса в системе $\mathrm{Mo} / \mathrm{Be}$ более выгодно образование на границе $\mathrm{MoBe}_{2}$, а не $\mathrm{MoBe}_{22}$. Рефлекса от чистого Мо не наблюдается.

У образца с толщиной $\sim 1 \mathrm{~nm}$ Мо в структуре наблюдаются рефлексы только от $\mathrm{MoBe}_{2}$ (рис. 8). Рефлексов от $\mathrm{MoBe}_{22}$ нет ни в исходном состоянии, ни после термического отжига. Однако наличие рефлексов при меньших толщинах слоя Мо подразумевает их образование в системе с достаточной толщиной для их обнаружения. И если их отсутствие после термического отжига было бы объяснимо переходом перемешанной зоны в более стабильное состояние - $\mathrm{MoBe}_{2}$, то полное отсутствие рефлексов $\mathrm{MoBe}_{22}$ при толщине Мо в структуре $\sim 1 \mathrm{~nm}$ непонятно. Рефлекса от чистого Мо не наблюдается.

У образцов с толщиной Мо $\sim 2.3 \mathrm{~nm}$, как известно из [13], наблюдаются только рефлексы от чистого Мо. Никаких рефлексов от бериллидов не наблюдается как в исходном состоянии, так и после термического отжига, несмотря на то, что их общая толщина на границах раздела должна составлять порядка $1 \mathrm{~nm}$ или более. Их отсутствие на картине с большой толщиной Мо в структуре пока не объяснено.

\section{Выводы и заключение}

По результатам поведенного комплексного исследования внутренней структуры Мо/Ве многослойных зеркал и ее динамики при вакуумном отжиге были получены следующие результаты. 
В структуре $\mathrm{Mo} / \mathrm{Be}$ на границах раздела образуются перемешанные слои разного состава. Бериллид $\mathrm{MoBe}_{22}$ образуется на границе Мо-на-Ве из-за большего количества атомов Ве, окружающих тяжелый Мо, который во время осаждения проникает вглубь Ве пленки, и $\mathrm{MoBe}_{2}$ на границе Ве-на-Мо из-за малой энергии частиц $\mathrm{Be}$, не проникающего в большом количестве в плотную пленку Мо.

В ходе термического отжига в течении $4 \mathrm{~h}$ соединение $\mathrm{MoBe}_{22}$ на границе Мо-на-Ве переходит в $\mathrm{MoBe}_{2}$. Это может быть объяснено следующим образом. Ве проникает глубже в Мо, чем Мо в Ве. В этом случае доля $\mathrm{Be}$ в пограничной области снижается за счет ушедших атомов. Соответственно возрастает доля бериллида с меньшим содержанием бериллия. На границе Ве-на-Мо заметных изменений не происходит, господствующим бериллидом здесь был до отжига и остается после него $\mathrm{MoBe}_{2}$.

Так как никаких структурных изменений в слоях в течение $1 \mathrm{~h}$ отжига при температуре $T=280^{\circ} \mathrm{C}$ не наблюдается, полученный результат подтверждает гипотезу, предложенную в [12], о механизме роста коэффициента отражения образцов при одночасовом отжиге в вакууме в области $11 \mathrm{~nm}$ за счет частичного вытеснения кислорода из бериллиевых слоев. При дальнейшем отжиге происходят сильные диффузионные процессы с образованием другого соединения на границе раздела, а также сильный рост уровня шероховатости межслоевых границ, которые вносят более сильный негативный вклад в оптические характеристики, чем вытеснение кислорода.

Остается не проясненным несоответствие выводов настоящей работы выводам работы [18], где утверждается, что соединение с большим количеством Ве образуется на границе Ве-на-Мо, а не наоборот. Данное несоответствие требует дополнительных исследований.

Также остается непонятным отсутствие следов соединений при большой толщине Мо в многослойной системе. Исходя из данных EXAFS-спектроскопии, можно сделать вывод, что толщина перемешанных зон на обеих границах составляет не менее $1 \mathrm{~nm}$, что много по сравнению со слоем Мо толщиной $2 \mathrm{~nm}$.

\section{Финансирование работы}

Работа выполнена в рамках выполнения государственного задания № 0035-2014-0204 и при поддержке грантов РФФИ № 19-32-90154, 19-02-00081, 18-02-00588, 1802-00173, - интерпретация экспериментальных данных EXAFS, с использованием оборудования ЦКП „Физика и технологии микро- и наноструктур“ при ИФМ РАН.

\section{Конфликт интересов}

Авторы заявляют, что у них нет конфликта интересов.

\section{Список литературы}

[1] Montcalm C., Bajt S., Mirkarimi P.B., Spiller E.A., Weber F.J., Folta J.A. // Proceedings. 1998. Vol. 3331. P. 42.

[2] Skulina K.M., Alford C.S., Bionta R.M., Makowiecki D.M., Gullikson E.M., Soufli R., Kortright J.B., Underwood J.H. // Appl. Opt. 1995. Vol. 34. P. 3727-3730.

[3] Mirkarimi P.B., Bajt S., Wall M.A. // Appl. Opt. 2000. Vol. 39. P. $1617-1625$.

[4] Singh M., Braat J.J.M. // Appl. Opt. 2000. Vol. 39. P. 2189 2197.

[5] Mirkarimi P.B. // Opt. Eng. 1999. Vol. 38. N 7. P. 1246-1259.

[6] Bajt S. // J. Vac. Sci. Technol. A. 2000. Vol. 18. N 2. P. $557-$ 559.

[7] Wu B., Kumar A. // Appl. Phys. Rev. 1. 2014. P. 011104.

[8] Chkhalo N.I., Salashchenko N.N. // AIP Advances. 2013. Vol. 3. P. 082130-1.

[9] Вайнер Ю.А., Гарахин С.А., Зуев С.Ю., Нечай А.Н., Плешков Р.С., Полковников В.Н., Салащенко Н.Н., Свечников М.В., Сертсу М.Г., Смертин Р.М., Соколов А., Чхало Н.И., Шаферс Ф. // УФН. 2020. Т. 190. С. 92-106.

[10] Svechnikov M.V., Chkhalo N.I., Gusev S.A., Nechay A.N., Pariev D.E., Pestov A.E., Polkovnikov V.N., Tatarskiy D.A., Salashchenko N.N., Schäfers F., Sertsu M.G., Sokolov A., Vainer Y.A., Zorina M.V. // Opt. Express. 2018. Vol. 26. N 26. P. 33718-33731.

[11] Chkhalo N., Gusev S., Nechay A., Pariev D., Polkovnikov V., Salashchenko N., Schäfers F., Serts M., Sokolov A., Svechnikov M., Tatarsky D. // Opt. Lett. 2017. Vol. 42. N 24. P. 5070-5073.

[12] Nechay A.N., Chkhalo N.I., Drozdov M.N., Garakhin S.A., Pariev D.E., Polkovnikov V.N., Salashchenko N.N., Svechnikov M.V., Vainer Yu.A., Meltchakov E., Delmotte F. // AIP Advances. 2018. Vol. 8. P. 075202.

[13] Смертин Р.М., Гарахин С.А., Зуев С.Ю., Нечай А.Н., Полковников Н.В., Салащенко Н.Н., Свечников М.В., Sertsu M.G., Sokolov A., Чхало Н.И., Schäfers F., Юнин П.А. // ЖТФ. 2019. Т. 89. Вып. 11. С. 1783-1788.

[14] Slaughter J.M., Kearney P.A., Schulze D.W., Falco C.M., Hills C.R., Saloman E.B., Watts R.N. // Proceedings. SPIE. 1991. Vol. 1343. P. 73-82.

[15] Rosen R.S., Stearns D.G., Viliardos M.A., Kassner M.E., Vernon S.P., Cheng Y. // Appl. Opt. 1993. Vol. 32. N 34. P. 6975-6980.

[16] Braun S., Mai H., Moss M., Scholz R., Leson A. // Jpn. J. Appl. Phys. 2002. Vol. 41. Pt 1. N 6B. P. 4074-4081.

[17] Sakhonenkov S.S., Filatova E.O., Gaisin A.U., Kasatikov S.A., Konashuk A.S., Pleshkov R.S., Chkhalo N.I. // Phys. Chem. Chem. Phys. 2019. Vol. 21. P. 25002-5010.

[18] Kasatikov S.A., Filatova E.O., Sakhonenkov S.S., Gaisin A.U., Polkovnikov V.N., Smertin R.M. // J. Phys. Chem. C. 2019. Vol. 123. N 42. P. 25747-25755.

[19] Самсонов Г.В. // Бериллиды. 1996. С. 45-47.

[20] Лякишев Н.П. // Диаграммы состояния двойных металлических систем. 1996. Т. 1. С. 587-589.

[21] Svechnikov M., Pariev D., Nechay A., Salashchenko N., Chkhalo N., Vainer Y., Gaman D. // J. Appl. Cryst. 2017. Vol. 50. P. $1428-1440$.

[22] Chernyshov A.A., Veligzhanin A.A., Zubavichus Y.V. // Nucl. Instrum. Methods Phys. Res. A. 2009. Vol. 603. N 1-2. P. 95-98. 
[23] Электронный ресурс. Режим доступа. http://kcsni.nrcki.ru/ pages/main/sync/beamlines/stm/index.shtml

[24] Ravel B., Newville M. // J. Synchr. Rad. 2005. Vol. 12. P. 537541.

[25] Zabinsky S.I., Rehr J.J., Ankudinov A., Albers R.C., Eller M.J. // Phys. Rev. 1995. Vol. 52. N 4. P. 2995-3009.

[26] Joly Y. // Phys. Rev. 2001. Vol. 63. N 12. P. 125120-125129.

[27] Guda S.A., Guda A.A., Soldatov M.A., Lomachenko K.A., Bugaev A.L., Lamberti C., Gawelda W., Bressler C., Smolentsev G., Soldatov A.V., Joly Y. // J. Chem. Theory Comp. 2015. Vol. 11. N 9. P. 4512-4521.

[28] Hedin L., Lundqvist B.I. // J. Phys. C. 1971. Vol. 4. N 14.

[29] Gates-Rector S., Blanton T. // Powder Diffr. 2019. Vol. 34. N 4. P. 352-360.

[30] Випа̌u O., Joly Y. // J. Phys. Cond. Mat. 2009. Vol. 21. N 34. P. 345501. 